\title{
Übelkeit und Erbrechen in der Schwangerschaft: Ingwer - eine wirksame Alternative?
}

\author{
Prof. Dr. Martin Smollich, Pharmakologe, Rheine
}

\section{Bei Schwangerschaftsübelkeit und -erbrechen steht als Wirkstoff der 1. Wahl das Anti- histaminikum Doxylamin zur Verfügung. Bei stärkeren Beschwerden gibt es außerdem zahlreiche weitere Wirkstoffe, die sicher eingesetzt werden können. Trotzdem wird - insbesondere bei leichteren Beschwerden - häufig nach pflanzlichen Alternativen gefragt. Am bekanntesten in diesem Zusammenhang ist die Anwendung von Ingwer.}

Die Ingwer-Pflanze (Zingiber officinale) ist eine ausdauernde Staude, die in den tropischen Regionen Südostasiens beheimatet ist [1]. Bereits in der Antike gelangte die Pflanze nach Europa, wo sie seither arzneilich genutzt wird. Dabei werden nicht die oberirdischen Teile der Pflanze verwendet, sondern der unterirdische Wurzelstock (Rhizom), den man unverarbeitet auch als Lebensmittel und Gewürz kennt. Zu den wichtigsten Inhaltsstoffen des Ingwer-Wurzelstocks gehören das aromatisch riechende ätherische Öl mit der Hauptkomponente $\alpha$-Zingiberen sowie die nicht-flüchtigen Scharfstoffe (Gingerole und Shogaole) [2]. Die Einnahme erfolgt entweder als Teezubereitung oder in Form von Kapseln, die den pulverisierten Ingwer-Wurzelstock enthalten.

Traditionell wird Ingwer zur Anregung der Magensaftproduktion und der gastrointestinalen Peristaltik angewendet sowie als mildes Antiemetikum. Grundlage dieser Effekte ist vermutlich die Interaktion von Ingwer-Inhaltsstoffen mit gastrointestinalen Muskarin- und Serotonin-Rezeptoren [3].

Die für die Bewertung von Phytopharmaka zuständige Kommission E bei der deutschen Arzneimittelzulassungsbehörde (BfArM) nennt als Anwendungsgebiete für Ingwer dyspeptische Beschwerden und die Prophylaxe von Reise- übelkeit; explizit als Kontraindikation genannt wird dagegen die Anwendung in der Schwangerschaft [4]. Allerdings stammt diese Beurteilung bereits aus dem Jahr 1990 und inzwischen sind zahlreiche Studien zur antiemetischen Wirksamkeit von Ingwer durchgeführt worden, insbesondere auch bei Schwangeren.

Die unübersichtliche und widersprüchliche Studienlage zur Wirksamkeit und Sicherheit von Ingwer-Anwendungen bei Schwangerschaftsübelkeit und -erbrechen wird in einer aktuellen Übersichtsarbeit aus dem Jahr 2014 sehr gut dargestellt [5]: Dabei wurden zwölf randomisiert-kontrollierte Studien aus den Jahren 1991 bis 2011 ausgewertet mit insgesamt 1.278 Teilnehmerinnen.

Zusammenfassend konnte gezeigt werden, dass Ingwer-Wurzelstock in Dosierungen zwischen 1-2 g/Tag die Schwangerschaftsübelkeit lindert, ohne jedoch die Häufigkeit von Erbrechen zu reduzieren, sofern dieses vorhanden war. Relevante Nebenwirkungen der IngwerAnwendung wurden nicht beobachtet. Interessanterweise stellt sich auch heraus, dass niedrigere Dosierungen (1$1,5 \mathrm{~g} / \mathrm{Tag})$ wirksamer waren als höhere Dosierungen (>2 g/Tag). Diese Einschätzungen werden auch durch ein aktuelles Cochrane-Review bestätigt [6].
Doch auch wenn es deutliche Hinweise darauf gibt, dass Ingwer bei Schwangerschaftsübelkeit und -erbrechen wirksam ist, so haben sämtliche Studien erhebliche Schwachpunkte [7]:

- In sämtlichen Untersuchungen wurde meist nur wenige Tage bis maximal drei Wochen therapiert, weshalb sich zur Wirksamkeit und auch zur Sicherheit einer längeren Anwendung keine verlässliche Aussage machen lässt.

- Außerdem wurde die Mehrzahl der Studien aus Sicherheitsgründen bei Schwangeren nach der 20. SSW durchgeführt, was nicht der tatsächlichen hohen Prävalenz von Übelkeit und Erbrechen zu Beginn der Schwangerschaft entspricht.

Auch wenn einiges dafür spricht, dass die Anwendung von Ingwer in der Schwangerschaft wirksam und sicher ist, so sind für eine valide wissenschaftliche Beurteilung geeignetere Studien erforderlich. Diese Einschätzung spiegelt sich auch darin wider, dass die Rote Liste Ingwer nach wie vor in die Schwangerschaftskategorie 5 einordnet („Ausreichende Erfahrungen über die Anwendung beim Menschen liegen nicht vor“), und dass im Beipackzettel von IngwerPräparaten die Anwendung in der Schwangerschaft nicht empfohlen wird. 


\section{Fazit}

Die aktuelle Studienlage deutet darauf hin, dass Ingwer-Wurzelstock bei leichteren Formen von Schwangerschaftsübelkeit und -erbrechen wirksam sein kann; in diesem Fall kann Ingwer als Tee oder in Kapselform probiert werden.

\section{Allerdings ist nur die kurzfristige Anwendung (bis zwei Wochen) in Dosierungen bis 1,5 g/Tag hinsichtlich einer möglichen embryonalen Toxizität nachweislich unbedenklich.}

Auf eine hochdosierte ( $>2 \mathrm{~g} / \mathrm{Tag}$ ) und auf die längerfristige Anwendung sollte verzichtet werden, da höhere Dosierungen vermutlich weniger wirksam sind und die Sicherheit zudem nicht belegt ist.

Nicht angewendet werden sollte Ingwer bei bestehendem Reflux oder Sodbrennen, da sich diese Beschwerden durch die enthaltenden Scharfstoffe verstärken können.

Außerdem sollte man bedenken, dass die Empfehlung von Ingwer-Präparaten bei Schwangeren zu Irritationen führen kann, da diese Präparate - einschließlich des bekannten Zintona ${ }^{\circledR}$ - ausschließlich für Reiseübelkeit zugelassen sind. Zudem ist im Beipackzettel der Hinweis enthalten, dass das Präparat in der Schwangerschaft sicherheitshalber nicht eingenommen werden soll.

Bleibt der Therapieversuch mit Ingwer ohne ausreichenden Erfolg, sollte rasch auf Doxylamin oder Vitamin $\mathrm{B}_{6}$ (ggf. auch in Kombination) gewechselt werden.

\section{Literatur}

1 Hänsel R, Sticher O (2003). Pharmakognosie - Phytopharmazie. 7. Auflage, Springer Heidelberg

2 Teuscher E et al. (2012). Biogene Arzneimittel: Lehrbuch der Pharmazeutischen Biologie. 7. Auflage, Wissenschaftliche Verlagsgesellschaft Stuttgart

3 Pertz HH et al. (2011). Effects of ginger constituents on the gastrointestinal tract: role of cholinergic $\mathrm{M} 3$ and serotonergic 5-HT3 and 5-HT4 receptors. Planta Med 77: 973-978

4 Kommission E (1988). Monographie Ingwerwurzelstock, ATC-Code: A04AF. Bundesanzeiger 85
5 Viljoen E et al. (2014). A systematic review and meta-analysis of the effect and safety of ginger in the treatment of pregnancy-associated nausea and vomiting. Nutr J 13: 20

6 Matthews A et al. (2014). Interventions for nausea and vomiting in early pregnancy. Cochrane Database Syst Rev 3: CD007575

7 Ding M et al. (2013). The effectiveness and safety of ginger for pregnancy-induced nausea and vomiting: a systematic review. Women Birth 26: e26-30

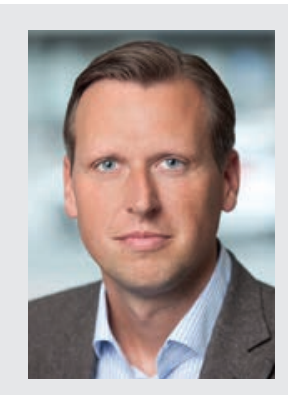

Prof. Dr. Martin Smollich Klinische Pharmakologie Mathias Hochschule Rheine Frankenburgstraße 31 48431 Rheine E-Mail: m.smollich@mhrheine.de 